\title{
THE GEOMETRIES ASSOCIATED WITH A CERTAIN SYSTEM OF
}

\section{CREMONA GROUPS*}

\author{
BY \\ JOHN WESLEY YOUNG AND FRANK MILLETT MORGAN
}

Introduction. A geometry is defined by a class of elements $S$ and a group of transformations $G$ operating in a one-to-one reciprocal way on the elements of $S$ and transforming $S$ into itself. The geometry $(S, G)$ then consists of such properties of figures (i. e., combinations of elements of $S$ ) as remain invariant under the transformations of $G$. This is in brief Klein's classical principle. $\dagger$

The most familiar geometries having the point as the primary element of space which have received systematic study are:

(1) The geometries defined by the general projective groups in one, two, . . . , $n$ dimensions, and their subgroups.

(2) The geometries defined by the inversion groups in two, three, and to some extent $n$ dimensions.

The object of the present paper is to call attention to a system of groups $G_{2}, G_{3}, \cdots, G_{n}$ of Cremona transformations in point spaces $S_{2}, S_{3}, \cdots, S_{n}$ of two, three, . ., $n$ dimensions and to develop elementary properties of the corresponding geometries. This system of groups includes for the case $n=2$ the group $G_{2}$ of direct circular transformations in the plane; the corresponding plane geometry is then closely related to the ordinary inversion geometry in the plane. The associated geometries in three or more dimensions form, therefore, a direct extension of the inversion geometry in a plane, different trom the inversion geometries in the spaces of three or more dimensions. The elementary curves in the geometry $\left(S_{2}, G_{2}\right)$ are the conics through two fixed points in a projective plane (in the ordinary orientation of the inversion geometry these points are the circular points at infinity so that the conics referred to are circles). In the geometries $\left(S_{3}, G_{3}\right)$ the elementary

* Presented to the Society, under different titles, by J. W. Young, December 28, 1911, and by J. W. Young and F. M. Morgan, April 26, 1913, and April 24, 1915.

$\dagger$ Felix Klein, Vergleichende Betrachtungen über neuere geometrische Forschungen, Erlangen, 1873. Reprinted Mathematis che Annalen, Vol. 43 (1892), pp. 63-100. English translation by M. W. Haskell, Bulletin of the American Mathemati c a 1 S o c i e ty, Vol. 2 (1893), pp. 215-49. 
curves are twisted cubics; in the geometries $\left(S_{n}, G_{n}\right)$ they are normal curves of order $n$ in $S_{n}$.

Definition of groups $G_{2}, G_{3}, \cdots, G_{n}$. It will be sufficient to define in detail the groups $G_{2}$ and $G_{3}$. The extension to $G_{n}$ will then be sufficiently obvious.

The Group $G_{2}$. Let $U_{1}, U_{2}$ be the distinct centers of two pencils of lines in the (reai or complex) projective plane $\Sigma_{2}$. Every point $P$ in the plane not on the line $U_{1} U_{2}$ determines and is determined by a unique line in each of the pencils $U_{1}, U_{2}$. The point $P$ is then determined by the two lines $l_{1}$ (through $U_{1}$ ) and $l_{2}$ (through $U_{2}$ ) and we may write $P=\left(l_{1}, l_{2}\right)$. Let $\pi_{1}$ be any projectivity in the pencil $U_{1}$ and $\pi_{2}$ any projectivity in the pencil $U_{2}$ and let $\pi_{1}\left(l_{1}\right)=l_{1}^{\prime}$ and $\pi_{2}\left(l_{2}\right)=l_{2}^{\prime}$. The transformation $\pi=\left(\pi_{1}, \pi_{2}\right)$ is then defined as the transformation which transforms $P=\left(l_{1}, l_{2}\right)$ into $P^{\prime}=\left(l_{1}^{\prime}, l_{2}^{\prime}\right) . \pi$ is a transformation of $G_{2}$, and $G_{2}$ consists of all such transformations $\pi$ which can be obtained by varying the component projectivities $\pi_{1}, \pi_{2}$. $G_{2}$ is a group of (in general) quadratic transformations which transforms into itself transitively the system of all conics through $U_{1}$ and $U_{2}$.

The Group $G_{3}$. Let $u_{1}, u_{2}, u_{3}$ be the nonconcurrent axes of three pencils of planes in projective space $\Sigma_{3}$. Every point $P$ in $\Sigma_{3}$ (not on the quadric surface determined by $u_{1}, u_{2}, u_{3}$ ) determines a unique plane in each of the pencils $u_{1}, u_{2}, u_{3}$. The point $P$ is then determined by the three planes $\alpha_{1}$ (through $u_{1}$ ), $\alpha_{2}$ (through $u_{2}$ ), $\alpha_{3}$ (through $u_{3}$ ) and we may write $P=\left(\alpha_{1}, \alpha_{2}, \alpha_{3}\right)$. Let $\pi_{1}$ be a projectivity in the pencil $u_{1}, \pi_{2}$ a projectivity in the pencil $u_{2}$, and $\pi_{3}$ a projectivity in the pencil $u_{3}$; and let $\pi_{i}\left(\alpha_{i}\right)=\alpha_{i}^{\prime}(i=1,2,3)$. The transformation $\pi=\left(\pi_{1}, \pi_{2}, \pi_{3}\right)$ is then defined as the transformation which transforms $P=\left(\alpha_{1}, \alpha_{2}, \alpha_{3}\right)$ into $P^{\prime}=\left(\alpha_{1}^{\prime}, \alpha_{2}^{\prime}, \alpha_{3}^{\prime}\right) . \pi$ is in general a cubic transformation and is a transformation of $G_{3} . G_{3}$ consists of all such transformations $\pi$ which can be obtained by varying the component projectivities $\pi_{1}, \pi_{2}, \pi_{3} . G_{3}$ transforms into itself transitively the system of all twisted cubics having $u_{1}, u_{2}, u_{3}$ as bisecants.

The group $G_{n}(n>3)$ is defined similarly in $\Sigma_{n}$ by means of projectivities in $n$ pencils of $\Sigma_{n-1}$ 's. They are groups of Cremona transformations of order $n$ (in general), which transform transitively into themselves certain systems of normai curves of order $n$ in $\Sigma_{n}$.

It should be noted that for $n>2, G_{n}$ has several distinct forms. Thus $G_{3}$ has four distinct forms according to the figure formed by the lines $u_{1}, u_{2}, u_{3}$. These lines may be skew to each other $\left(G_{3}^{1}\right)$, or two only may intersect $\left(G_{3}^{2}\right)$, or one of the lines may intersect each of the other two without their being coplanar $\left(G_{3}^{3}\right)$, or finally $u_{1}, u_{2}, u_{3}$ may form a triangle $\left(G_{3}^{4}\right)$. The cubic transformations thus defined have been extensively studied by Noether and 
others. * The groups of such transformations have, however, received but little attention hitherto. $\dagger$

In what follows explicit reference is generally made only to the case $n=3$. Most of our theorems, however, apply either without any change or with very obvious changes to all cases, $n \geqq 2$. The following notational scheme enables us readily to assign the proper generality to the later results. For the case $n=2$ there is only one group $G_{2}$ while for the case $n=3$ there are four groups $G_{3}^{1}, G_{3}^{2}, G_{3}^{3}, G_{3}^{4}$ : the symbol $G_{3}$ is used to represent any one of these last four groups. Similarly $G_{n}$ represents any one of the groups possible for a general $n$; while, when the number of dimensions is not significant, we shall simply use $G$ to represent any one of the whole system of groups.

The space $S$. The group $G_{3}$ has been defined in terms of, and as acting on, the (real or complex) projective point space $\Sigma_{3}$ of three dimensions. It does not, however, operate on the points of $\Sigma_{3}$ in a one-to-one reciprocal way. Our first problem is, then, to define a space $S_{3}$, on the primary elements of which $G_{3}$ operates in a one-to-one reciprocal way. The definition of such a space is very simple, for we need only keep in mind the fact that $G_{3}$ does operate in a reciprocally one-to-one way on the triples of planes $\alpha_{1}, \alpha_{2}, \alpha_{3}$. We may therefore simply define these plane-triples as the "points" of our space $S_{3} . \ddagger$ However for the sake of greater intuitional satisfaction, we prefer to replace these plane triples by "points," proper and improper, determined by them in a one-to-one reciprocal way.

We have first as proper points of $S_{3}$, all the points of $\Sigma_{3}$ which are not on the quadric surface $\left(u_{1} u_{2} u_{3}\right) . \S$

We bave secondly, improper points, in general as follows:

(1) Straight lines when the planes of the plane-triple $\left(\alpha_{1}, \alpha_{2}, \alpha_{3}\right)$ intersect in a line. These lines are the lines meeting $u_{1}, u_{2}$, and $u_{3}$.

(2) Point-planes, consisting of a point on $u_{i}(i=1,2,3)$ with a plane through $u_{i}$. These occur when two of the planes $\left(\alpha_{1}, \alpha_{2}, \alpha_{3}\right)$ meet on $u_{i}$.

* Noether, Über die eindeutigen Raumtransformationen, insbesondere in ihrer Anwendung auf die Abbildung algebraischer Flächen, $\mathrm{M}$ a th e mat is c he A n $\mathrm{n}$ al e n, Bd. 3 (1871), S. 547-580.

Doehlemann, Über eine einfache eindeutige Raumtransformation dritter Ordnung, M ü n c he ner Beri c h t e, Bd. 24 (1894), S. 41-50.

Doehlemann, Geometrische Transformationen, Teil (2), S. 308.

Sturm, Die Lehre von den Geometrischen Verwandtschaften, IV, §127.

† Fano, I gruppi di Jonquières generalizzati, T or i n o M e mori e (2), vol. 48 (1898), p. 221.

Hudson, The $(3,3)$ Transformations in Space, Proce edings of the London Mathematical Society, 1913.

$\ddagger$ For $G_{n}$ we should define the "points" of $S_{n}$ as the corresponding $n$-ples of $\Sigma_{n-1}$ 's.

$\S$ This quadric degenerates in the cases $G_{3}^{2}, G_{3}^{3}$ into two planes; in case of $G_{3}^{4}$ into a single plane. 
When two of the lines $u_{1}, u_{2}, u_{3}$ intersect, other forms of improper points arise as will be seen by a direct consideration of the cases in question.

To define completely the spaces $S_{3}^{1}, S_{3}^{2}, S_{3}^{3}, S_{3}^{4}$ associated respectively with $G_{3}^{1}, G_{3}^{2}, G_{3}^{3}, G_{3}^{4}$, it is only necessary to list the improper points associated in each case with the proper points to form the space $S_{3}$ in question. We give below such a definition for $S_{3}^{1}$ because of its simplicity, and for $S_{3}^{4}$ as it is in some respects the most interesting.

The space $S_{3}^{1}$ of $G_{3}^{1}$. (The lines $u_{1}, u_{2}, u_{3}$ are skew to each other.)

(1) Proper points: All the points of the projective $\Sigma_{3}$ not on the quadric $\left(u_{1} u_{2} u_{3}\right)$.

(2) Improper points:

(i) All the lines meeting $u_{1}, u_{2}, u_{3}$.

(ii) All the point-planes consisting of a point $P$ on $u_{i}(i=1,2,3)$ together with any plane through $u_{i}$ distinct from the tangent plane to the quadric $\left(u_{1} u_{2} u_{3}\right)$ at $P$.

If the projective $\Sigma_{3}$ is real, there are $\infty^{1}$ improper points of the first kind, and three systems of $\infty^{2}$ improper points of the second kind.

The space $S_{3}^{4}$ of $G_{3}^{4}$. (The lines $u_{1}, u_{2}, u_{3}$ form a triangle.)

(1) Proper points: Ali points of the projective $\Sigma_{3}$ not on the plane of the triangle.

(2) Improper points:

(i) All linear elements consisting of a vertex $U_{i}(i=1,2,3)$ of the triangle $u_{1} u_{2} u_{3}$ and a line through $U_{i}$, not in the plane of $u_{1}, u_{2}, u_{3}$.

(ii) All line-planes consisting of a line $u_{i}(i=1,2,3)$ and any plane through $u_{i}$ distinct from the plane of $u_{1} u_{2} u_{3}$.

(iii) The plane of the triangle $u_{1} u_{2} u_{3}$.

If $S_{3}$ is real, there are three systems of $\infty^{2}$ improper points of the first kind, three systems of $\infty^{1}$ improper points of the second kind, and one improper point of the third kind.

Throughout the remainder of the paper unless otherwise specified the word "point" will refer to an element, proper or improper, of the space $S$ associated with the group $G$.

Analytic representation of $G$. The associated hyper-complex number system. If we establish a projective scale in each of the pencils $u_{i}(i=1,2,3)$ by assigning the numbers $0,1, \infty$ to any three distinct planes through $u_{i}$, ${ }^{*}$ then every point $P$ in $S_{3}$ may be denoted by three coördinates $\left(x_{1}, x_{2}, x_{3}\right)$, $x_{i}$ being the coördinate of the plane through $P$ in $u_{i}$ in the scale just referred to. The transformations of the group $G_{3}$ are then given by the relations:

$$
x_{i}^{\prime}=\frac{a_{i} x_{i}+b_{i}}{c_{i} x_{i}+d_{i}}, \quad a_{i} d_{i}-b_{i} c_{i} \neq 0 \quad \quad(i=1,2,3) .
$$

* Veblen and Young, Projective Geometry, Vol. 1, Boston, 1910, p. 141. 
If $A=\left(a_{1}, a_{2}, a_{3}\right)$ and $B=\left(b_{1}, b_{2}, b_{3}\right)$ are any two points of $S_{3}$ (neither of which is on a plane $\infty$ ) a hypercomplex algebra $N_{3}{ }^{*}$ is defined by the relations,

$$
\begin{aligned}
A+B & =\left(a_{1}+b_{1}, a_{2}+b_{2}, a_{3}+b_{3}\right), \\
A \cdot B & =\left(a_{1} b_{1}, a_{2} b_{2}, a_{3} b_{3}\right) .
\end{aligned}
$$

It is at once evident from this definition that $N_{3}$ satisfies the ordinary associative, commutative, and distributive laws as to addition and multiplication. $\dagger$ The nil-factors (divisors of zero) are the elements $\left(x_{1}, x_{2}, x_{3}\right)$ in which one or more of the $x_{i}(i=1,2,3)$ are zero. Moreover it should be noted that the elements of the form $(x, x, x)$ form a sub-algebra $R_{3}$ of $N_{3}$ isomorphic with the algebra of ordinary real or complex numbers according as $x$ ranges over the system of real or complex numbers. The numbers of $R_{3}$ may be conveniently designated as the scalars of $N_{3}$.

In the terms of this algebra, the transformations of $G_{3}$ are given by the relations:

$$
X^{\prime}=\frac{A X+B}{C X+D} \quad(A D-B C \neq \text { a nil factor }),
$$

where $A, B, C, D$ represent any elements of $N_{3}$.

The extension of these results to any group $G_{n}$ is obvious. $\ddagger$

Invariant of $G$. Cross-ratio. Associated with every ordered set of four points

$$
\begin{array}{ll}
A=\left(\alpha_{1}, \alpha_{2}, \alpha_{3}\right), & B=\left(\beta_{1}, \beta_{2}, \beta_{3}\right), \\
C=\left(\gamma_{1}, \gamma_{2}, \gamma_{3}\right), & D=\left(\delta_{1}, \delta_{2}, \delta_{3}\right)
\end{array}
$$

are three cross-ratios $\rho_{i}=\mathrm{R}_{\psi}\left(\alpha_{i} \beta_{i}, \gamma_{i} \delta_{i}\right)(i=1,2,3)$. The cross ratio symbol $\rho=\left(\rho_{1}, \rho_{2}, \rho_{3}\right)=\mathbf{R}_{\psi}(A B, C D)$ is then invariant under $G_{3}$, and is hence fundamental in the geometry $\left(S_{3}, G_{3}\right)$. It will be noted that the cross-ratio symbol $\rho$ is an element of the hypercomplex algebra $N_{3}$. This close connection of $(S, G) \S$ with the projective geometry in a one-dimensional

* In papers by J. W. Young, A New Formulation for General Algebra, read before the American Mathematical Society, Dec. 28, 1911, and Apr. 26, 1913 (abstracts in B ullet in, vol. 18, Feb., 1912; vol. 19, July, 1913), the groups here discussed were discovered as a natural result of the investigations of these papers.

$\dagger$ The algebra $N_{3}$ is not invariant under $G$, that is to say if $A+B=C$, and if an operation of the group sends $A, B, C$ into $A^{\prime}, B^{\prime}, C^{\prime}$ then, in general, $A^{\prime}+B^{\prime} \neq C^{\prime}$.

$\ddagger$ The representation of $G$ in the form (3) exhibits strikingly the analogy of $G$ with the group $G_{2}$ of direct circular transformations in a plane. The corresponding algebra $N_{2}$ is equivalent to the algebra of ordinary complex numbers if properly orientated in the real $S_{2}$. The representation of the real circular transformations in a plane by linear fractional transformations on a complex variable is well known.

$\$$ Reference may be made to a paper by J. W. Young, The Geometry of Chains on a Complex Line, A n a ls of $\mathrm{M}$ a the mat i c s, series 2, vol. 11, pp. 33-48, 1909-10. The methods and the results of this paper and the present one bear a close analogy. Indeed, the geometries $(S, G)$ are in a sense geometries on a hypercomplex line. 
form at once suggests a method of approach to the geometry $(S, G)$. Certain conceptions and the associated terminology of one-dimensional projective geometry may at once be carried over. For example, two pairs of points $A B, C D$ of $S_{3}$ form a harmonic set $H(A B, C D)$ if the sets of planes $\left(\alpha_{i} \beta_{i}\right.$, $\left.\gamma_{i} \delta_{i}\right)(i=1,2,3)$ are harmonic: i. e., if $\mathrm{R}(A B, C D)=(-1,-1,-1)$. It is natural to designate a scalar $(x, x, x)$ of $N_{3}$ by the number $x$. With this notation we may say that $A B, C D$ form a harmonic set if

$$
\mathbf{R}(A B, C D)=-1 \text {. }
$$

The fundamental theorem of $G$. Let us call two points $A=\left(\alpha_{1}, \alpha_{2}, \alpha_{3}\right)$ and $A^{\prime}=\left(\alpha_{1}^{\prime}, \alpha_{2}^{\prime}, \alpha_{3}^{\prime}\right)$ of $S_{3}$ co-axial if they determine the same plane in any one of the pencils $u_{i}$, i. e., if either $\alpha_{1}=\alpha_{1}^{\prime}$, or $\alpha_{2}=\alpha_{2}^{\prime}$, or $\alpha_{3}=\alpha_{3}^{\prime}$.*

Theorem 1. If $A, B, C$ and $A^{\prime}, B^{\prime}, C^{\prime}$ are any two sets of three points of $S$, such that no two points of either set are co-axial, then there exists one and only one transformation $\pi$ of $G$, such that $\pi(A B C)=A^{\prime} B^{\prime} C^{\prime}$.

This theorem is a direct consequence of the definition of $G$ and the fundamental theorem of projective geometry.

The simple curves of $(S, G)$. Let $A, B, C$ be any three points of $S_{3}$ no two of which are co-axial, and consider the locus of a point $P$ such that $\mathrm{R},(A B, C P)$ is a scalar $\lambda=(\lambda, \lambda, \lambda)$ of $N_{3}$, where $\lambda$ is any real or complex variable. This locus may also be defined as the locus of points determined by the triples of homologous planes in the projectivities

$$
\alpha_{1} \beta_{1} \gamma_{1} \pi \alpha_{2} \beta_{2} \gamma_{2} \pi \alpha_{3} \beta_{3} \gamma_{3} .
$$

A curve thus defined we will call a $C$-curve. In view of the following theorems, which are immediate consequences of the definition and Theorem 1, the $C$-curves are simple one dimensional elements of the geometry $(S, G)$.

Theorem 2. If $A, B, C$ are any three points of $S$, no two of which are co-axial, there exists one and only one $C$-curve containing $A, B$, and $C$.

The $C$-curve through $A, B, C$ will be denoted by $|A B C|$.

Theorem 3. Any two $C$-curves are equivalent under the group $G$.

The $C$-curves in $S_{3}$ are, in general, twisted cubics (in $\Sigma_{3}$ ) having the axes $u_{1}, u_{2}, u_{3}$ as bisecants and any such twisted cubic is a $C$-curve. $\dagger$ However

${ }^{*}$ In $S_{n}, A=\left(\alpha_{1}, \alpha_{2}, \alpha_{3}, \cdots \alpha_{n}\right)$ and $A^{\prime}=\left(\alpha_{1}^{\prime}, \alpha_{2}^{\prime}, \alpha_{3}^{\prime}, \cdots \alpha_{n}^{\prime}\right)$ are co-axial if one of the relations $\alpha_{i}=\alpha_{i}^{\prime}$ holds.

† That the locus of the point of intersection of the homologous planes of three projective axial pencils is in general a twisted cubic having the axes of the pencils as bisecants, seems to have been first proved by Chasles, Comptes Rendus, vol. 45 (1857), p. 189 and Journal de Mathématiques, 2d ser., vol. 2 (1857), p. 397. See also Reye, Geometrie der Lage, vol. 2, pp. 91-93; Sturm, Geometrische Verwandtschaften, vol. I, p. 306. In $S_{n}$ the $C$-curves are normal curves of order $n$ in $\Sigma_{n}$, having as $(n-1)$-secants the "axes" of the generating pencils of $\Sigma_{n-1}$ 's, and every such normal curve is a $C$-curve: see Bertini, Introd. alla geometria proiettiva degli iperspazi, p. 275. 
these cubics degenerate under certain conditions into conics and straight lines. It is quite unnecessary for the further development of this paper, to describe these curves in detail; for, all later theorems are consequences merely of the definitions and Theorems 1, 2, 3. It seems desirable nevertheless to list the $C$-curves completely, at least for $S_{3}^{1}$ and $S_{3}^{4}$.

The class of $C$-curves in $S_{3}^{1}$ consist of: (1) All the twisted cubics of $\Sigma_{3}$ having $u_{1}, u_{2}, u_{3}$ as bisecants. These are characterized in $S_{3}^{1}$ by the fact that none of them contains an improper point of the first kind. (2) All the conics of $\Sigma_{3}$ not on the quadric $u_{1} u_{2} u_{3}$ but meeting $u_{1}, u_{2}$, and $u_{3}$ each once. These are characterized in $S_{3}^{1}$ by the fact that each such $C$-curve contains one and only one improper point of the first kind. (3) All the straight lines of $\Sigma_{3}$ not on the quadric $u_{1} u_{2} u_{3}$ nor meeting $u_{1}, u_{2}, u_{3}$. These are characterized by the fact that each such $C$-curve contains two and only two distinct improper points of the first kind. (4) The locus of all improper points of the first kind.

The class of $C$-curves for $S_{3}^{4}$ consists of: (1) All twisted cubics of $\Sigma_{3}$ containing the vertices $U_{1}, U_{2}, U_{3}$ of the triangle $u_{1}, u_{2}, u_{3}$. These are characterized in $S_{3}^{4}$ by the fact that every such $C$-curve contains three improper points of the first kind and no other improper points. (2) All conics of $\Sigma_{3}$ not on the plane $u_{1}, u_{2}, u_{3}$ but passing through $U_{i}(i=1,2,3)$ and meeting the opposite side of the triangle. These are characterized in $S_{3}^{4}$ by the fact that each such conic contains an improper point of the first kind, one of the second kind and no other improper points. (3) All straight lines of $S_{3}$ not through a side of the triangle $u_{1} u_{2} u_{3}$. These are characterized in $S_{3}$ by the fact that each such $C$-curve contains the improper point of the third kind and no other improper point.*

A fundamental property of a $C$-curve, which follows at once from the definition, is given by

Theorem 4. If $A, B, C$ are three distinct points of a $C$-curve, the harmonic conjugate of $A$ with respect to $B$ and $C$ is on the curve.

Properties of $G$. The projective structure of the group $G$ makes it possible to infer numerous properties of the group almost immediately. The enumeration of subgroups and their properties, questions of the conjugacy of trans-

* Metric orientation. In the usual orientation of the real geometry $\left(S_{2}, G_{2}\right)$ the two points $U_{1} U_{2}$ coincide with the circular points at infinity in the plane $\Sigma_{2}$. The system of $C$-curves (the conics through $U_{1}, U_{2}$ ) then appear as the system of circles (and straight lines) in $S_{2}$. An analogous orientation of the geometry $\left(S_{3}^{4}, G_{3}^{4}\right)$ in real $\Sigma_{3}$ may be obtained by letting the triangle $u_{1}, u_{2}, u_{3}$ consist of two conjugate tangents to the absolute conic at infinity and their real chord of contact. If $U_{1}$ be the real intersection of these tangents, and if we choose the direction determined by $U_{1}$ to be vertical, the system of real $C$-curves consists of (1) The twisted cubics on all right circular cylinders whose axes are vertical. (2) The rectangular hyperbolas one of whose asymptotes is vertical. (3) The straight lines which are neither vertical nor horizontal.

Trans Am. Math. Soc. 16 
formations, etc., while simple, we pass over as not of immediate concern in the present paper. The following results are needed, however, in what follows.

ThEOREM 5. Every transformation of $G$ transforms a harmonic set of points into a harmonic set of points.

Theorem 6. Two transformations of $G$ with the same double points are commutative.

The involutions, $I$, of $G$ (i. e., transformations $I$ such that $I^{2}=1, I \neq 1$ ) are of special interest. Since every component involution in the generating pencils has two distinct double planes in (complex) $\Sigma_{3}$, it is clear that every involution $I$ of $G_{3}$ has in general $2^{3}=8$ double points in complex $S_{3}$. These eight double points consist of four pairs, the two points of each pair not being co-axial. Such a pair of non-co-axial double points will be called a pair of conjugate double points* of $I$ and will be denoted by $M_{i} N_{i}(i=1,2,3,4) . \dagger$ We have then,

Theorem 7. In an involution $I$ of $G$ any point $A$ and its image $A^{\prime}$ are harmonic with respect to any pair of conjugate double points $M_{i} N_{i}$ of $I$. Symbolically $I(A)=A^{\prime}=H\left(A, M_{i} N_{i}\right)$.

TheOREM 8. An involution $I$ of $G$ is completely determined by two pairs of homologous points; and, in particular, by a pair of conjugate double points.

Theorem 9. Any transformation $\pi$ of $G$ is the product of two involutions of $G$.

Theorem 10. If $A, A^{\prime}$ is a pair of homologous points of an involution $I$, the involution having $A, A^{\prime}$ for double points is commutative with $I$.

CoRollary. The product of two involutions with conjugate double points $A, A^{\prime}$ and $B, B^{\prime}$, respectively, transforms into itself the involution in which $A, A^{\prime}$ and $B, B^{\prime}$ are homologous pairs.

Invariant Curves. It will be supposed in what follows that $S$ is complex.

Theorem 11. If a $C$-curve through $M_{i} N_{i}$ and one through $M_{j} N_{j}(i \neq j)$ [ $M_{i} N_{i}$ and $M_{j} N_{j}$ being conjugate double points of an involution $\left.I\right]$ have one point in common they have a second point in common.

Let $C$ and $C^{\prime}$ be the $C$-curves and let $P$ be their common point.

Since the harmonic conjugate of $P$ is on both $C$ and $C^{\prime}$ the curves have a second point in common.

Theorem 12. Every C-curve containing a pair of conjugate double points $M_{i} N_{i}$ of an involution $I$ is invariant under $I$.

Let $C$ be any $C$-curve containing the double points $M_{i} N_{i}$ and let $A$ be any

* An involution $I$ of $G_{n}$ has in general $2^{n}$ double points in (complex) $S_{n}$ consisting of $2^{n-1}$ pairs of non-co-axial points, i. e., conjugate double points.

† Intuitionally, the situation is clarified by thinking of the eight double points as the eight corners of a box. Conjugate double points are diagonally opposite corners. 
point of $C=\left|M_{i} N_{i} A\right|$. Then $I(A)=A^{\prime}=H\left(A, M_{i} N_{i}\right)$. Hence $A^{\prime}$ is a point of $C$.

THEOREM 13. Every C-curve invariant under an involution I passes through a pair of conjugate double points of $I$.

CoRollary. Through every point in $S_{3}$ distinct from $M_{i} N_{i}(i=1,2,3,4)$ pass four and only four $C$-curves invariant under $I$.

Theorem 14. If a $C$-curve $C$ not passing through a given pair of conjugate points $M_{i} N_{i}$ of an involution I meets two $C$-curves through $M_{i} N_{i}$ in two pairs of points harmonic with regard to $M_{i} N_{i}$ then $C$ passes through a pair of double points $M_{j} N_{j}(i \neq j)$.

Let $A, A^{\prime}$ and $B, B^{\prime}$ be the two pairs of points. Then $H\left(M_{i} N_{i}, A A^{\prime}\right)$ and $H\left(M_{i} N_{i}, B B^{\prime}\right)$ gives $M_{i} N_{i}$ as the double points of the involution $I\left(A A^{\prime}, B B^{\prime}\right)$. Therefore $\left|A A^{\prime} B\right|=\left|A^{\prime} A B^{\prime}\right|$ which is an invariant $C$-curve and thus by Theorem 12 must pass through the points $M_{j} N_{j}$.

THEOREM 15. If some $C$-curve is left invariant by a non-involutoric transformation $\pi$ of $G$, then there is one and only one $C$-curve invariant under $\pi$ through every point $P$ of $S$ not co-axial with a double point of $\pi$.

Let $C$ be the $C$-curve invariant under $\pi$, and let $A$ be any point of $C$ not a double point of $\pi$. Let $\pi_{1}$ be the transformation of $G$ having the same double points as $\pi$ and such that $\pi(A)=P$. Let $\pi_{1}(C)=C^{\prime}$. Then

$$
\pi_{1} \pi \pi_{1}^{-1}\left(C^{\prime}\right)=C^{\prime}
$$

but $\pi_{1} \pi \pi_{1}^{-1}=\pi$, therefore $\pi\left(C^{\prime}\right)=C^{\prime}$, which proves that there is at least one $C$-curve through $P$ invariant under $\pi$. Now suppose that there are two distinct $C$-curves through $P$ invariant under $\pi$ and let

$$
\pi(P)=P^{\prime}, \quad \pi\left(P^{\prime}\right)=P^{\prime \prime} .
$$

The curve $C^{\prime}$ being invariant, gives $P, P^{\prime}, P^{\prime \prime}$ as points of $C^{\prime}$. If $P^{\prime \prime} \neq P$ these three points determine $C^{\prime}$ uniquely: if $P^{\prime \prime}=P, \pi$ is an involution and this contradicts the hypothesis.

THEOREM 16. If the transformation $\pi$ of $G$ is parabolic (i. e., has only one double point) then through every point in $S$ non-co-axial with the double point, passes one and only one C-curve invariant under $\pi$ and every such curve passes through the double point.

Let $A$ be any point in $S$ and $\pi(A)=A^{\prime}, \pi\left(A^{\prime}\right)=A^{\prime \prime}$. Then, if $M$ is the double point, $\left(M A^{\prime}, A A^{\prime \prime}\right)$ is a harmonic set. But $\pi\left(A A^{\prime} M\right)=A^{\prime} A^{\prime \prime} M$. Therefore, there is a $C$-curve through $A$ invariant under $\pi$. That there is only one follows from Theorem 15.

Tangent C-Curves. Two $C$-curves are said to be tangent to each other provided there exists a parabolic transformation $\pi$ of $G$ which leaves them 
both invariant.* Two such $C$-curves will have only one point $M$ in common (Theorem 16). The point $M$ is called the point of contact.

The relation of tangency is clearly an invariant of the geometry $[S, G]$.

Theorem 17. If $M$ is a point on a $C$-curve $C$ and $P$ any point non-co-axial with $M$ and not on $C$, then there exists one and only one $C$-curve through $P$ and tangent to $C$ at $M$.

For let $\pi$ be any parabolic transformation of $G$ with double point $M$, and let $\pi(P)=P^{\prime} . \quad\left|P P^{\prime} M\right|$ is then the $C$-curve sought.

Orthogonal $C$-Curves. A pair of non-co-axial points $M, N$ of $S_{3}$ determines uniquely three other pairs of non-co-axial points, namely the other three pairs of conjugate double points of the involution of which $M, N$ are one pair of conjugate double points. In real $S_{3}$ if $M, N$ are real, the other three pairs may all be imaginary. It seems convenient, therefore, to introduce the following terminology derived by obvious analogy from the geometry $\left(S_{2}, G_{2}\right)$.

A $C$-curve $C$ is said to be about two non-co-axial points $M, N$, if it passes through the points of one of the three pairs of non-co-axial points determined by $M, N$. The points $M, N$ are then said to be conjugate with respect to $C$.

The relation here defined between a $C$-curve and certain pairs of points is clearly an invariant under $G_{3}$, as expressed by the following theorem:

Theorem 18. If a $C$-curve $C$ is transformed by a transformation $\pi$ of $G$ into $C^{\prime}$, any pair of points conjugate with respect to $C$ is transformed by $\pi$ into a pair of points conjugate with respect to $C^{\prime}$.

A $C$-curve $C_{2}$ is said to be orthogonal to a $C$-curve $C_{1}$, if $C_{2}$ contains two points conjugate to $C_{1} \cdot \dagger$

Theorem 19. If $C_{2}$ is orthogonal to $C_{1}, C_{1}$ is orthogonal to $C_{2}$.

* Two $C$-curves tangent to each other as here defined are tangent in the ordinary sense, and conversely. For the coördinate system described on p. 236 may be so chosen that any point $M$ is $(0,0,0)$ and that any $C$-curve through $M$ is given by $x=y=z$. Any other $C$-curve through $M$ is then given by the equations

$$
\frac{a x}{c x+d}=\frac{a^{\prime} y}{c^{\prime} y+d^{\prime}}=\frac{a^{\prime \prime} z}{c^{\prime \prime} z+d^{\prime \prime}}, \quad a a^{\prime} a^{\prime \prime} d d^{\prime} d^{\prime \prime} \neq 0 \text {. }
$$

The conditions that these intersect in a second point $T=(t, t, t)(t \neq 0)$ are

$$
\frac{a}{c t+d}=\frac{a^{\prime}}{c^{\prime} t+d^{\prime}}=\frac{d^{\prime \prime}}{c^{\prime \prime} t+d^{\prime \prime}}
$$

and that they be tangent in the ordinary sense at $M(t=0)$ are

$$
\frac{a}{d}=\frac{a^{\prime}}{d^{\prime}}=\frac{a^{\prime \prime}}{d^{\prime \prime}}
$$

But these are precisely the conditions that (1) can be obtained from $x=y=z$ by a parabolic transformation with double point $M$.

$\dagger$ It should be noted that orthogonality, as here defined, is not in general equivalent to orthogonality in the ordinary sense. 
Theorem 20. Any pair of orthogonal C-curves is transformed by a transformation of $G$ into a pair of orthogonal $C$-curves.

This theorem establishes orthogonality as an invariant of $G$.

THEOREM 21. If a transfcrmation $\pi$ of $G$ leaves each of two orthogonal C-curves invariant, $\pi$ is an involution.

This follows from the proof of Theorem 15.

Theorem 22. Through two distinct points $A, B$ on a $C$-curve $C$ in $S_{3}$, pass three and only three $C$-curves orthogonal to $C$.

For, let $M, N$ be any pair of points on $C$ harmonic with $A, B$ : and let $M_{i} N_{i}(i=1,2,3)$ be the three other pairs of double points of the involution $I$ having $M, N$ for double points. Then we have $H\left(M_{i} N_{i}, A B\right)$ and the three curves $\left|A B M_{i}\right|(i=1,2,3)$ are orthogonal to $C$. That no other $C$-curves orthogonal to $C$ pass through $A, B$ will follow, if we show that the three $C$-curves orthogonal to $C$ obtained as above from any other two points $M^{\prime}, N^{\prime}$ of $C$, harmonic with $A B$, coincide with those obtained from $M, N$. To this end we will show that if $A B M M^{\prime}$ are on $C$, then $A B M_{1} M_{1}^{\prime}$ will be on the same $C$-curve. The following notational scheme is used:

$$
\begin{array}{lll}
M=\left(u_{1}, u_{2}, u_{3}\right), & N=\left(v_{1}, v_{2}, v_{3}\right), & M^{\prime}=\left(u_{1}^{\prime}, u_{2}^{\prime}, u_{3}^{\prime}\right), \\
N^{\prime}=\left(v_{1}^{\prime}, v_{2}^{\prime}, v_{3}^{\prime}\right), & A=\left(\alpha_{1}, \alpha_{2}, \alpha_{3}\right), & B=\left(\beta_{1}, \beta_{2}, \beta_{3}\right):
\end{array}
$$

finally $M_{1}=\left(v_{1}, u_{2}, u_{3}\right)$ and $M_{1}^{\prime}=\left(v_{1}^{\prime}, u_{2}, u_{3}\right)$. The condition that $A, B, M, M^{\prime}$ are on $C$ is

$$
\mathbf{R}\left(\alpha_{1} \beta_{1}, u_{1} u_{1}^{\prime}\right)=\mathbf{R}_{\psi}\left(\alpha_{2} \beta_{2}, u_{2} u_{2}^{\prime}\right)=\mathbf{R}\left(\alpha_{3} \beta_{3}, u_{3} u_{3}^{\prime}\right) .
$$

We must show that

$$
\mathbf{R}\left(\alpha_{1} \beta_{1}, v_{1} v_{1}^{\prime}\right)=\mathbf{R}\left(\alpha_{2} \beta_{2}, u_{2} u_{2}^{\prime}\right)=\mathbf{R}_{\psi}\left(\alpha_{3} \beta_{3}, u_{3} u_{3}^{\prime}\right)
$$

or simply that

$$
\mathrm{R}\left(\alpha_{1} \beta_{1}, u_{1} u_{1}^{\prime}\right)=\mathrm{R}\left(\alpha_{1} \beta_{1}, v_{1} v_{1}^{\prime}\right) .
$$

But this equality follows directly from the relations $H\left(\alpha_{1} \beta_{1}, u_{1} v_{1}\right)$ and $H\left(\alpha_{1} \beta_{1}, u_{1}^{\prime} v_{1}^{\prime}\right)$. The theorem is clearly valid in any geometry $(S, G)$, if the number of $C$-curves through $A, B$ orthogonal to $C$ is replaced by $2^{n-1}-1$.

Orthogonal Systems of $C$-Curves. It is now natural to generalize the wellknown orthogonal systems of circles in $\left(S_{2}, G_{2}\right)$. These are of two kinds. (1) The system of all circles through two distinct points $A, B$ and the system of all circles about the two points $A, B$. (2) The system of all circles tangent at a point $M$ to a given circle through $M$ and the system of all circles tangent at $M$ to a circle through $M$ orthogonal to a circle of the first system. 
In $\left(S_{3}, G_{3}\right)$ we have analogously:

Theorem 23. Two non-co-axial points $M, N$ of $S_{3}$ determine four systems of $C$-curves, viz., the system of all $C$-curves through $M, N$ and the three systems of $C$-curves about $M, N$. Any $C$-curve of one of these four systems is orthogonal to every $C$-curve of the other three systems.

TheOREM 24. Given four mutually orthogonal C-curves $C_{1}, C_{2}, C_{3}, C_{4}$ through $M, P$. The four systems of $C$-curves consisting of all the $C$-curves tangent to $C_{i}(i=1,2,3,4)$ at $M$ are such that any $C$-curve of one system is orthogonal to every $C$-curve of the other three systems.

In $\left(S_{n}, G_{n}\right)$, analogous theorems hold, the number of mutually orthogonal systems of $C$-curves being $2^{n-1}$.

Dartmodth College 